\title{
Capacitive Emulation for LCL-Filtered Grid-Connected Converters
}

\author{
Jose Miguel Espi (D), Rafael Garcia-Gil * (D) and Jaime Castello (iD \\ Department of Electrical Engineering, University of Valencia, Avd. Universitat S/N, 46100 Burjassot-Valencia, \\ Spain; Jose.M.Espi@uv.es (J.M.E.); jaime.castello@uv.es (J.C.) \\ * Correspondence: Rafael.garcia-gil@uv.es; Tel.: +34-963-544-023
}

Academic Editor: Frede Blaabjerg

Received: 1 June 2017; Accepted: 3 July 2017; Published: 5 July 2017

\begin{abstract}
This paper proposes an effective capacitive emulation (CE) technique to reduce the grid current distortion when the converter currents are controlled (converter current feedback (CCF)) in grid-connected converters with an LCL filter. Although the CCF scheme is preferable to the grid current feedback (GCF) scheme in terms of stability and inherent current limitation of the power semiconductors, the former presents a problem of additional grid-current harmonic distortion due to the LCL capacitor, which generates a distorted current by line voltage differentiation. To solve this problem, the distorting capacitive current is reproduced on the converter output, so that both cancel out each other on the grid. That is, the converter emulates a negative capacitance while delivering the active and reactive power at the fundamental frequency. This is achieved by adding an estimation of the distorting capacitive current to the converter current reference. Moreover, an effective CE technique requires a current control capable of tracking all harmonics added to the current reference and compensating for any control delay. To compensate this delay, the paper proposes a buffer-based method to advance and filter the current reference. The effectiveness of the CE method has been tested on a 10-kVA transformerless inverter with CCF and a simple proportional-integral (PI) control with grid-voltage feedforward cancellation. Experimental results prove that the proposed solution reduces the line current THD (total harmonic distortion) compared with the GCF strategy with proportional-resonant (PR) control.
\end{abstract}

Keywords: capacitive emulation (CE); converter current feedback (CCF); proportional resonant (PR) controller; total harmonic distortion (THD); grid-voltage feedforward

\section{Introduction}

The importance of the contribution of renewable energies to electric power generation is growing, the three-phase power converters playing a very important role. For these converters, the use of an LCL filter is preferred over an L filter, as the former can provide much less ripple and more harmonic attenuation using smaller passive elements to comply with standards such us IEEE 519-1992 and IEC 61000-3-12. However, the inherent resonance of the LCL filter can produce closed-loop instability [1-4].

When using LCL filters in grid-connected power converters, two common control strategies are used [4-8]: to sense and control the converter currents, i.e., converter current feedback (CCF) or to sense and control the grid currents directly, i.e., grid current feedback (GCF). The CCF has the advantage of an inherent semiconductor current limiting, with a straightforward over-current protection and a simpler power layout, whereas the over-current protection in the GCF case implies the use of current sensors at both the grid and converter sides.

However, when using CCF, even if the controlled inverter currents are sinusoidal, when grid voltages are distorted, grid currents are distorted, as well. The distortion mechanism is essentially 
as follows: the distorted grid voltage is applied to the LCL capacitors, which generate currents proportional to the time-derivative of the applied voltages and, hence, amplifying each voltage harmonic proportionally to its frequency. Finally, the distorted capacitor currents are added to the inverter currents to form the distorted grid currents, making the CCF solution worse than the GCF in terms of grid current quality.

As an attempt to overcome the distortion problem of the CCF, the GCF has recently emerged [9-12]. It is usually implemented using proportional-integral (PI) controls with the feedforward of the grid-voltage or proportional-resonant (PR) controls with enough bandwidth and the number of resonances to ensure rejection of the grid current harmonics. However, due to the higher resonance peak and phase drop in the GCF loop compared with the CCF loop [2,7], if acceptable control bandwidths are to be achieved, the resonance peak has to be strongly damped in GCF schemes. To avoid filter losses, this significant damping is usually carried out by means of active damping (AD) techniques, which basically consists of a proportional feedforward of the LCL capacitors currents $[9,10,13-17]$. This increases hardware or software complexity, either if more sensors are used to measure the capacitors currents or if the currents are estimated instead. In contrast, the CCF can achieve a high bandwidth and good stability margins with just a light passive damping, without the need for AD. Hence, the CCF is preferable also in terms of control simplicity.

With the aim to solve the problem of the grid current distortion while keeping the CCF advantages, this paper proposes a capacitive emulation (CE) technique. It consists of adding the distorted capacitor currents to the controlled inverter currents, so that both distorted capacitor and inverter currents cancel each other out on the grid. This method requires an accurate estimation of the grid-induced capacitor currents, to be added to the inverter current reference, and a control strategy capable of tracking all harmonics of the capacitor currents. A simple PI control with grid-voltage feedforward cancellation is used in this paper, which presents a variable group-delay between two and five samples in closed loop, as will be experimentally shown. Thus, this paper proposes also a buffer-based method to get a leading and filtered estimation that compensates for the delay introduced by the control. The filtering method is non-distorting, adapts to grid voltage amplitude and frequency variations and avoids peak currents when a grid voltage sag occurs.

Finally, the paper shows experimentally the effectiveness of the CE technique to reduce the THD of the grid currents in a 10-kVA transformerless grid-connected inverter with CCF and a PI control with grid-voltage feedforward cancellation. The THD (total harmonic distortion) results are compared with those obtained in the same inverter when using GCF and a proportional-resonant (PR) control, showing that with the proposed CCF + CE solution, the output current quality is improved while preserving the advantages of the CCF.

\section{Control Scheme}

In this section, the current control strategy of the three-phase grid-connected inverter of Figure 1 when regulating the grid currents (GCF) or the converter currents $(\mathrm{CCF})$ is described in the discrete-time domain considering control delays.

The LCL filter in Figure 1 can be represented by two decoupled circuits in the $\alpha \beta$ frame as shown in Figure $2 \mathrm{a}$, where $v_{g}$ and $v_{i}$ are the grid voltage and inverter averaged voltage, respectively. The inductors $Z_{1}$ and $Z_{2}$ are modeled with their inductances $L$ and series resistances $r$. The capacitor series resistance and any possible damping resistance are collected in $r_{c}$. Figure $2 \mathrm{~b}$ shows the equivalent block diagram of this filter. 


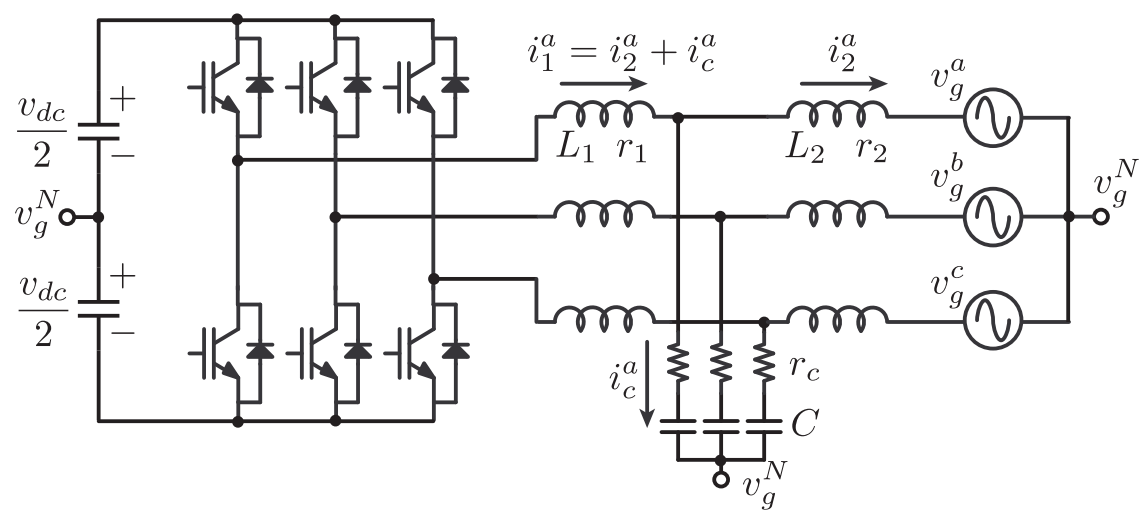

Figure 1. Transformerless three-phase grid-connected inverter with the LCL filter.

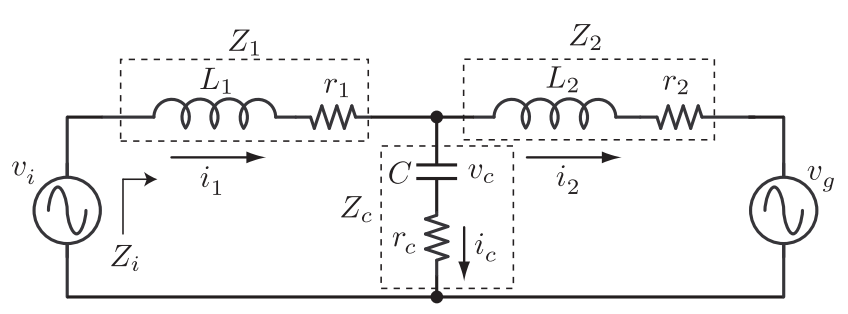

(a)

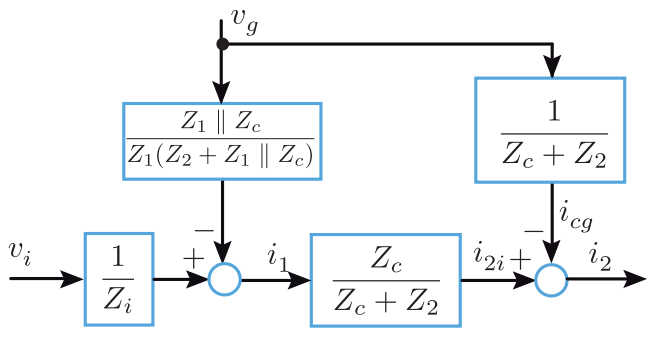

(b)

Figure 2. LCL filter model in the $\alpha \beta$ reference frame: (a) circuital and (b) block diagram model.

From Figure 2, the transfer functions from the converter output voltage $v_{i}$ to its output current $i_{1}$ or to the grid current $i_{2}$ are:

$$
\begin{gathered}
G_{i_{1}}=\frac{i_{1}(s)}{v_{i_{c}}(s)}=\frac{1}{Z_{i}} \approx \frac{s^{2}+C \omega_{o}^{2} r_{c} s+\omega_{o}^{2}}{L_{1} s\left(s^{2}+C \omega_{r}^{2} r_{c} s+\omega_{r}^{2}\right)} \\
G_{i_{2}}=\frac{i_{2}(s)}{v_{i_{c}}(s)}=\frac{1}{Z_{i}} \cdot \frac{Z_{c}}{Z_{c}+Z_{2}} \approx \frac{C \omega_{o}^{2} r_{c}\left(s+\frac{1}{r_{c} C}\right)}{L_{1} s\left(s^{2}+C \omega_{r}^{2} r_{c} s+\omega_{r}^{2}\right)}
\end{gathered}
$$

being $\omega_{o}=\frac{1}{\sqrt{L_{2} C}}$ and $\omega_{r}=\frac{1}{\sqrt{\left(L_{1} \| L_{2}\right) C}}$, where the inductor parasitic resistances $r_{1}$ and $r_{2}$ are neglected for worst case analysis and only $r_{c}$ including any passive damping component is considered.

In the case of (1), as $\omega_{r}$ approach $\omega_{0}$, by taking $L_{1} \gg L_{2}$, a nearly pole-zero cancellation can be achieved, reducing the resonance effect, as will be shown in the open-loop gain. This near cancellation or natural attenuation of the resonance is not possible in the case of GCF as shown in Equation (2).

At low frequencies, below $\omega_{1}=\frac{1}{\sqrt{L_{1} C}}<\omega_{0}<\omega_{r}$ yields $Z_{1} \| Z_{c} \approx Z_{1}$ and $Z_{2} \| Z_{c} \approx Z_{2}$, and the block diagram of Figure $2 \mathrm{~b}$ can be simplified by the power stage included in Figure 3, showing that the LCL filter behaves as an equivalent $L$ filter with inductance $L=L_{1}+L_{2}$ and series resistance $r_{L}=r_{1}+r_{2}$.

Figure 3 shows an overview of the complete control structure including power plant, anti-aliasing filters, controller and control delay. Switches (1), (2) and (3) represents programming options. With Switch (1), either a GCF or a CCF scheme may be chosen. The grid-voltage feedforward option can be selected according to Switch (2), and Switch (3) adds/removes the CE technique to the control. 


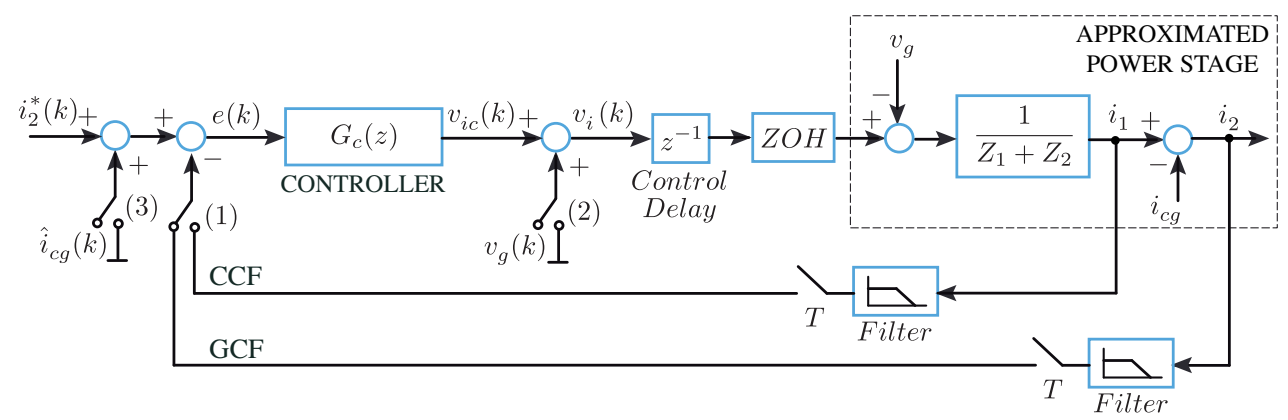

Figure 3. Plant model approximation for low frequencies with anti-aliasing filter, controller and control delay.

The control is formulated in the $d q$ reference frame, where inductors and capacitors are transformed as shown in Figure 4. Hence, decoupling terms ( $-L \omega_{g} i_{1}^{q}$ for variable $d$ and $L \omega_{g} i_{1}^{d}$ for variable $q$ ) must be added to the controller output in order to decouple the $d$ and $q$ current dynamics. These decoupling terms are assumed to be already included in the output signal of the controller $v_{i c}$, making the $\alpha \beta$ control diagram of Figure 3 fully equivalent in the $d q$ frame.

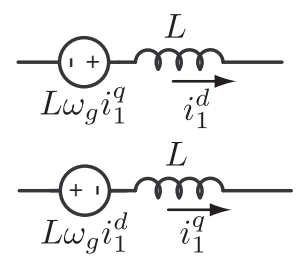

(a)

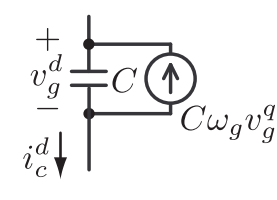

(b)

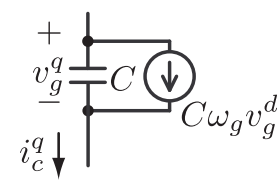

Figure 4. Inductor (a) and capacitor (b) in the $d q$ reference frame.

For the CCF scheme, a simple PI control with grid-voltage feedforward cancellation is considered. This cancellation is performed adding $v_{g}$ to the controller output by turning the Switch (2) ON in Figure 3. The PI compensator in discretized form is,

$$
G_{c}(z)=k_{p}+\frac{k_{i} T z}{z-1}
$$

where the proportional gain $k_{p} \approx L \omega_{c}$ sets the bandwidth, and the integral gain is $k_{i}=k_{p} \omega_{z}$, designed to set the PI's zero $\omega_{z}$ one decade below the bandwidth.

For the GCF scheme, a proportional-resonant (PR) controller has been used, which provides high gains at certain frequencies (resonant frequencies) eliminating steady state errors at these frequencies $[18,19]$. The PR controller, in the discretized form, is constructed by adding resonant compensators $G_{R_{h}}(z)$ in parallel to the previous PI controller,

$$
G_{c}(z)=k_{p}+\frac{k_{i} T z}{z-1}+\sum_{h} G_{R_{h}}(z)
$$

Each resonant compensator $G_{R_{h}}(z)$ is expressed in terms of an all-pass filter $G_{A P}(z)$ as [20]:

$$
G_{R_{h}}(z)=\frac{G_{h}}{2}\left[1-G_{A P_{h}}(z)\right]
$$

being $h$ the harmonic number, $G_{h}$ the resonant gain and:

$$
G_{A P_{h}}(z)=\frac{k_{2} z^{2}+k_{1}\left(1+k_{2}\right) z+1}{z^{2}+k_{1}\left(1+k_{2}\right) z+k_{2}}
$$




$$
\begin{array}{r}
k_{1}=-\cos \left(\omega_{h} T\right) \\
k_{2}=\frac{1-\tan \left(B W_{h} T / 2\right)}{1+\tan \left(B W_{h} T / 2\right)}
\end{array}
$$

where $\omega_{h}=h \omega_{g}$ is the resonant frequency and $B W_{h}$ is the resonant bandwidth.

Another important practical issue that must be taken into account in the analysis are the delays in the control loop that can significantly reduce the stability margins. In general [21], these delays are due to sensing circuits (anti-aliasing filters of the inverter currents), computational delays (time between the acquisition instant and the total update instant of the control calculations) and the pulse-width modulator (PWM) delay [18]. In this paper, the anti-aliasing filters have been designed to have a nearly constant group delay, so that they can be modeled as a pure delay term. Computational delays include sampling time and control calculations. In our case, a total control delay of $1.5 T_{S}$ has been considered.

Multiplying Equation (1) in discretized form with the PI controller (3) for CCF, multiplying the discretized form of (2) with the PR controller (4) for GCF and adding the control delay of $1.5 T_{S}$, the open-loop transfer functions of both feedback schemes are obtained.

For simulation and experimental results, the LCL filter used is specified in Table 1. The damping coefficient $\zeta=0.74$ of the anti-aliasing filters gives minimum variation of the group delay [22], minimizing the distortion of acquired currents. The programmed PI and PR control parameters are listed in Table 2, where a high bandwidth $\left(f_{c}=600 \mathrm{~Hz}\right)$ has been selected to track the highest number of grid voltage harmonics as possible.

Table 1. Plant parameters.

\begin{tabular}{lcc}
\hline Description & Variable & Value \\
\hline Nominal power & $P$ & $10 \mathrm{kVA}$ \\
Grid frequency & $\omega_{g}$ & $2 \pi 50 \mathrm{rad} / \mathrm{s}$ \\
Grid voltage (per line) & $v_{g}$ & $230 \mathrm{~V}_{\mathrm{rms}}$ \\
Switching frequency & $f_{s w}$ & $10 \mathrm{kHz}$ \\
DC-bus voltage & $v_{d c}$ & $800 \mathrm{~V}$ \\
Inverter side inductance & $L_{1}$ & $1.6 \mathrm{mH}$ \\
Inverter side series resistance & $r_{1}$ & $30 \mathrm{~m} \Omega$ \\
Grid side inductance & $L_{2}$ & $180 \mu \mathrm{H}$ \\
Grid side series resistance & $r_{2}$ & $120 \mathrm{~m} \Omega$ \\
LCL capacitance & $C$ & $19 \mu \mathrm{F}$ \\
Damping resistance & $r_{c}$ & $0.5 \Omega$ \\
Anti-aliasing filter (cutoff freq.) & $f_{a}$ & $5 \mathrm{kHz}$ \\
Anti-aliasing filter (damping) & $\zeta$ & 0.74 \\
\hline
\end{tabular}

Table 2. PI and PR control parameters.

\begin{tabular}{lcc}
\hline Description & Variable & Value \\
\hline Sampling period & $T$ & $50 \mu \mathrm{s}$ \\
Gain crossover frequency & $\omega_{c}$ & $2 \pi 600 \mathrm{rad} / \mathrm{s}$ \\
Proportional gain & $k_{p}$ & $6.71 \Omega$ \\
Integral gain & $k_{i}$ & $2530 \Omega \cdot \mathrm{s}^{-1}$ \\
2nd dq-harmonic resonant gain & $G_{2}$ & $40 \Omega$ \\
2nd dq-harmonic resonant bandwidth & $B W_{2}$ & $\pi \mathrm{rad} / \mathrm{s}$ \\
6th dq-harmonic resonant gain & $G_{6}$ & $60 \Omega$ \\
6th dq-harmonic resonant bandwidth & $B W_{6}$ & $2 \pi \mathrm{rad} / \mathrm{s}$ \\
12th dq-harmonic resonant gain & $G_{12}$ & $50 \Omega$ \\
12th dq-harmonic resonant bandwidth & $B W_{12}$ & $4 \pi \mathrm{rad} / \mathrm{s}$ \\
\hline
\end{tabular}

The open-loop Bode diagrams for GCF and CCF strategies obtained from MATLAB are shown in Figure 5, giving a gain and phase margins of $5.73 \mathrm{~dB}$ and $41.6^{\circ}$, respectively, for the GCF and $9.9 \mathrm{~dB}$ and $53.3^{\circ}$ for the CCF. The closed-loop step response is shown in Figure 6 giving a better response in the CCF case, in accordance with the obtained stability margins and the above explanation. 


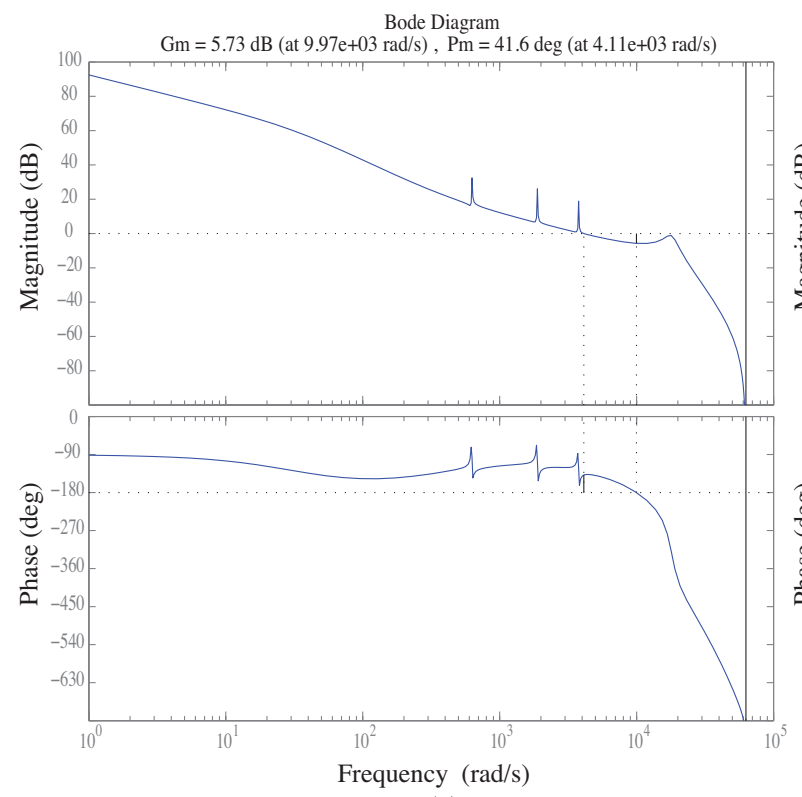

(a)

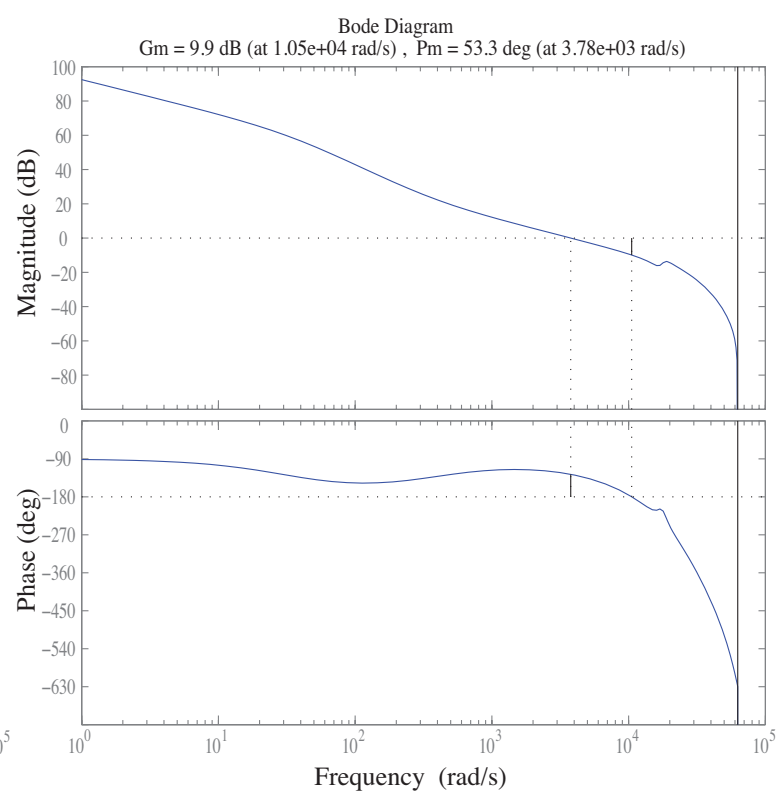

(b)

Figure 5. Open-loop bode diagram for Grid Current Feedback (GCF) (a) and CCF (b) strategy.

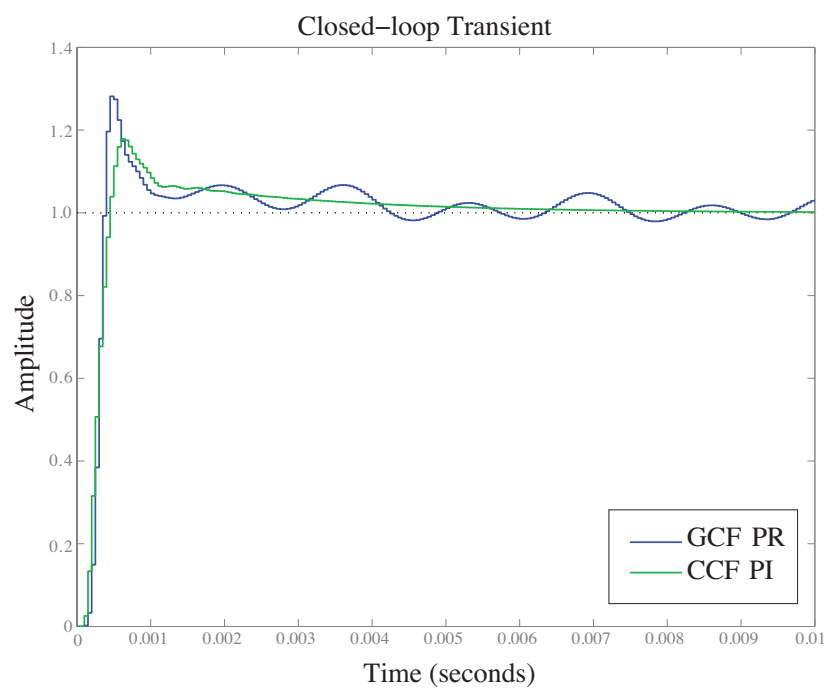

Figure 6. Step response for the GCF and CCF strategy. PR, Proportional-Resonant.

\section{Principles and Limitations of the Capacitive Emulation}

Figure 7 shows the simplified closed-loop CCF scheme in the $\alpha \beta$ reference frame. We assume the converter can generate current harmonics on $i_{1}$ in a given bandwidth, while $v_{g}$ shows all of its harmonic content. By the superposition principle, the line current $i_{2}$ is the sum of that generated by $i_{1}$ with a short-circuited grid, named $i_{2 i}$ in Figure $2 \mathrm{~b}$, and that generated by the grid voltage when the converter is switched off $\left(i_{1}=0\right)$, named $i_{c g}$ in Figure $2 \mathrm{~b}$. This second term is the distorting capacitive current, the main distortion source affecting the grid current when using CCF with LCL filters. From this analysis, we can affirm that:

1. The grid voltage harmonics below the resonance $L_{2}-C$ are applied over the LCL capacitors without attenuation, generating current distortion by capacitive differentiation. The grid voltage harmonics around the resonance can be amplified if no damping method (active or passive) is implemented. 
2. Only the generated harmonics on $i_{1}$ below the resonance $L_{2}-C$ are fed to the grid, and therefore, only these harmonics can cancel the distorting capacitive current on the grid.

3. The capacitor current harmonics below the resonance $L_{2}-C$ (including the fundamental) are distorting capacitive harmonics.

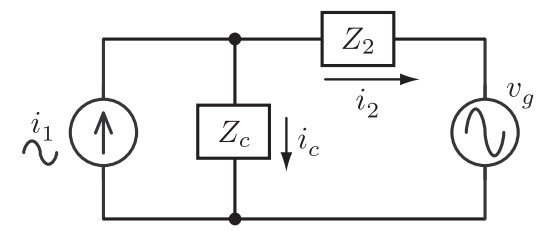

Figure 7. Closed-loop scheme with CCF in the $\{\alpha, \beta\}$ frame.

Hence, the CE method can cancel, at best, the distorting harmonics up to the $L_{2}-C$ resonance. Within this frequency interval, the distorting capacitive current matches the capacitor current and can be expressed in the $d q$ frame as (see Figure 4)

$$
\begin{aligned}
& i_{c g}^{d} \approx C\left(-v_{g}^{q} \omega_{g}+\frac{d v_{g}^{d}}{d t}\right) \\
& i_{c g}^{q} \approx C\left(v_{g}^{d} \omega_{g}+\frac{d v_{g}^{q}}{d t}\right)
\end{aligned}
$$

where $\omega_{g}$ is the grid angular frequency and $v_{c}^{d q} \approx v_{g}^{d q}$.

The proposed CE method consists of adding an estimation of the distorting current $i_{c g} d q$ to the inverter current reference (Figure 3):

$$
i^{* d q} \equiv i_{1}^{* d q}=i_{2}^{* d q}+i_{c g}^{d q}
$$

This estimation has to be ahead to compensate for the closed-loop delay of the current control.

Next, it will be shown that the CE technique requires a grid-voltage feedforward cancellation. In Figure 3, with Switch (1) in CCF mode, Switch (2) OFF and Switch (3) ON, the current harmonic tracking error in the $\alpha \beta$ frame yields:

$$
e_{1} \approx \frac{i_{1}^{*}+\frac{v_{g}}{Z_{1}+Z_{2}}}{1+H}
$$

where $H$ is the loop-gain represented in Figure $5 \mathrm{~b}$ and $i_{1}^{*}=i_{2}^{*}+\hat{i}_{c g}$, being $i_{2}^{*}$ the current reference for the fundamental frequency. If a perfect grid-voltage feedforward is performed, $v_{g}$ in Equation (10) is zero. Without grid-voltage feedforward, the contribution of $v_{g}$ to the error can be much higher than the contribution of the reference $i_{1}^{*}$. For instance, if we have a grid-voltage distortion of $1.6 \%$ for the fifth harmonic $\left(v_{g}(5)=0.16 \times 230 \mathrm{~V}_{e f f}\right)$, the distorting capacitor current is $i_{1}^{*}=\hat{i}_{c g}=5 C \omega_{g} v_{g}(5)=17.5 \mathrm{~mA}_{e f f}$ and $\frac{v_{g}}{Z_{1}+Z_{2}} \approx \frac{v_{g}(5)}{\left(L_{1}+L_{2}\right) 5 \omega_{g}}=8.3 \mathrm{~A}_{e f f}$. That is, the current error is much higher when grid-voltage feedforward is not used. In general, (10) can be written as $\left|e_{1}\right|=i_{1}^{*} \cdot|F(\omega)|$ where:

$$
|F(\omega)| \approx \frac{\left|1-p_{F F} \cdot\left(\frac{\omega_{12}}{\omega}\right)^{2}\right|}{|1+H(j \omega)|}
$$

being $\omega_{12}=\frac{1}{\sqrt{\left(L_{1}+L_{2}\right) C}}<\omega_{1}$, and $p_{F F} \in[0,1]$ indicates the amplitude of the feedforward residue ( $p_{F F}=0$ represents a perfect feedforward, and $p_{F F}=1$ represents the lack of feedforward). Figure 8 represents $|F|(\%)$ as a function of the harmonic number below the crossover frequency (13th harmonic) for $p_{F F}=0$ and $p_{F F}=1$, which indicates that the grid-voltage feedforward is necessary for the CE method to compensate the harmonics up to the crossover frequency. However, this is not a drawback, 
since the acquisition of the grid voltages is necessary for grid synchronization and for calculating the current reference of the $\mathrm{CE}$ method.

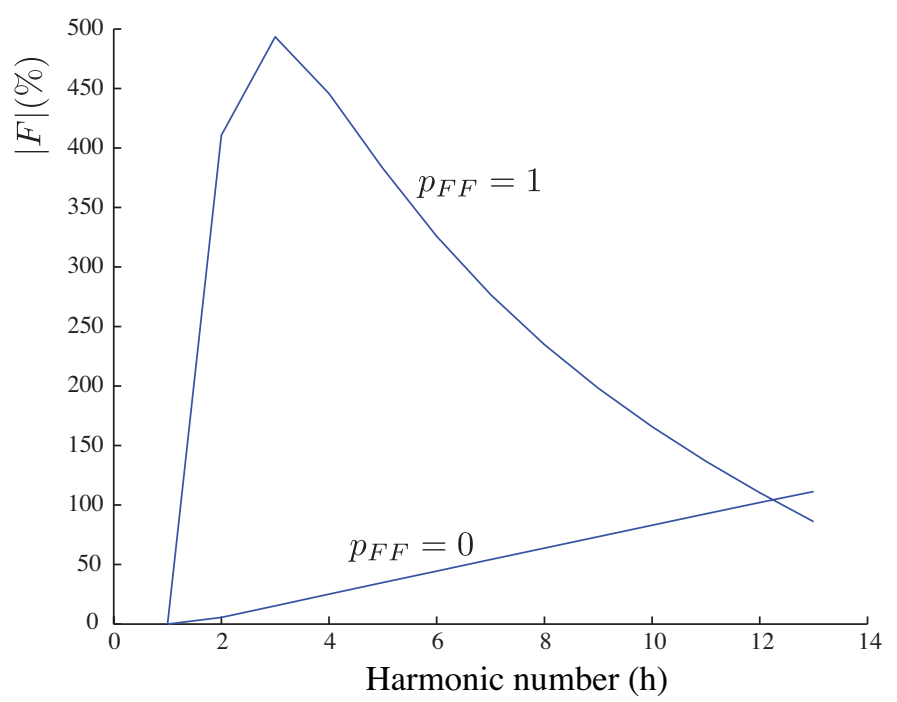

Figure 8. Current error relative to the current reference, as a function of the harmonic number, when using $\left(p_{F F}=0\right)$ or not $\left(p_{F F}=1\right)$ grid-voltage feedforward.

In case of weak networks, with a relatively large $L_{2}$, the attenuation of the switching harmonics is facilitated by this bigger inductance, whereby a smaller capacitor $C$ is normally installed in the LCL filter. Thus, the control can maintain the same bandwidth, and the CE method can still be applied, although it is less necessary because the harmonics generated by the capacitor are of a smaller amplitude.

\section{Distorting Current Estimation}

The distorting capacitor current estimation $\stackrel{i}{i} d q$, in the $d q$ reference frame, can be calculated as indicated by (8). This estimation has the advantage of not requiring additional sensors, but it contains derivative terms that generate noise and that have to be filtered. Moreover, this estimation has to be ahead to compensate for the current control delay (between two and five sampling periods), the voltage sensing delay and the reference calculation delay.

We propose to implement this derivative with a small phase lag (a group delay of approximately 0.6 sampling periods) by:

$$
\frac{d v_{g}(z)}{v_{g}(z)}=\left(\frac{0.8798}{T}\right) \frac{(z-1)}{z-0.1202}
$$

where $d v_{g}$ is the discrete derivative of $v_{g}$. This differentiator has been obtained by Tustin discretization of the system $\frac{s}{(2 T / \pi) \cdot s+1}$, which presents a pole at half the Nyquist frequency.

According to (8), the distorting current in the $d q$ frame can be calculated as:

$$
\begin{aligned}
& \hat{i}_{c g}^{d}=C\left(-v_{g}^{q} \omega_{g}+d v_{g}^{d}\right) \\
& \hat{i}_{c g}^{q}=C\left(v_{g}^{d} \omega_{g}+d v_{g}^{q}\right) .
\end{aligned}
$$

To filter this noisy estimation, a non-distorting buffer-based technique is proposed. Two constant-size arrays (for $d$ and $q$ coordinates) store the $N_{b}=2 \pi /\left(\omega_{g} T\right)=400$ filtered values of the estimation $<\hat{i}_{c g}>$. Each noisy estimation $\hat{i}_{c g}$ calculated using (13) is averaged with the filtered value stored at the writing index $k_{w}$ position: 


$$
<\hat{i}_{c g}>\left(k_{w}, k\right)=a \cdot<\hat{i}_{c g}>\left(k_{w}, k-1\right)+(1-a) \cdot \hat{i}_{c g}(k)
$$

where $0<a<1, k_{w}=$ round $\left(\frac{N_{b} \cdot \theta}{2 \pi}\right) \leq N_{b}$ and $\theta$ is the grid voltage angle given by a phase-locked loop (PLL). This is a very simple programming algorithm and offers a first order filtering for each of the 400 array positions. As it filters each array position instead of the whole signal, the result is a waveform without harmonic distortion. Values for $a$ around 0.9 give satisfactory filtering and adaptation to grid frequency variations. Moreover, the filtering removes any peak generated by (13) when a voltage sag occurs.

A leading estimation $n_{f}$ samples ahead is achieved by reading at the index position $k_{r}=\operatorname{rem}\left(k_{w}+\Delta k, N_{b}\right)$, being:

$$
\Delta k=\operatorname{round}\left(N_{b} \cdot n_{f} \cdot T \cdot \frac{\omega_{g}}{2 \pi}\right)
$$

where the function $\operatorname{rem}()$ is the remainder after division, and $\omega_{g}$ is obtained by means of a frequency-locked loop (FLL) algorithm [23]. Figure 9 shows, in the $a b c$ frame, the filtered distorting current estimation when the converter is switched off and when it operates at nominal power. An exaggerated number of advance samples $n_{f}=50$ has been programmed to better appreciate the shifting effect.

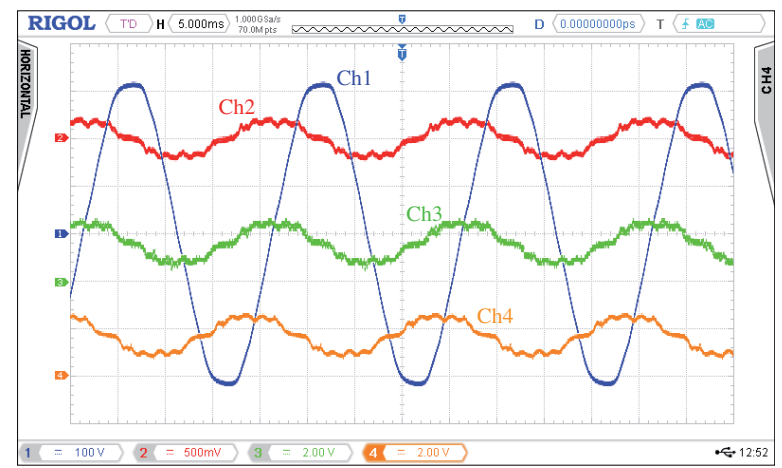

(a)

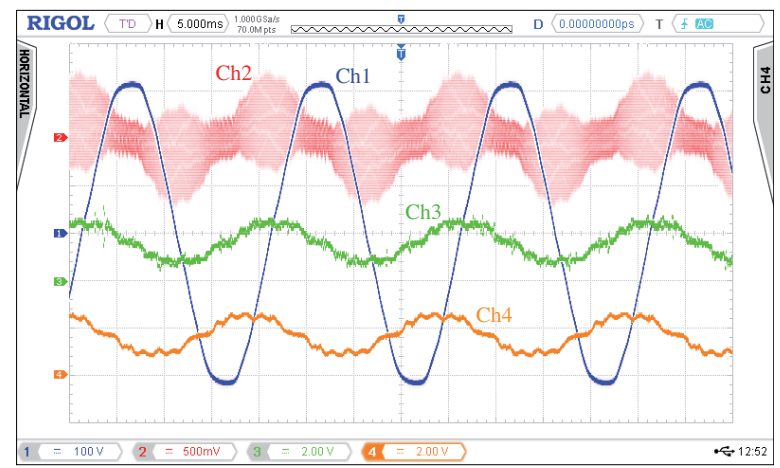

(b)

Figure 9. Distorting current estimation when the converter is switched off (a) and when operating at nominal power (b). Shown are: the grid voltage (Ch1), the current of the LCL capacitor (Ch2: 5A/div), the distorting current estimation without filtering (Ch3) and the filtered estimation 50 samples ahead (Ch4).

\section{Experimental Results}

A 10-kVA transformerless back-to-back converter was built to assess the CE strategy when using CCF and to compare it with a GCF strategy. The rectifier regulates the DC-bus voltage to $800 \mathrm{~V}$, while the inverter solves the current control and the CE method. Two intelligent power modules IPM-PS22A79 from Mitsubishi (Tokyo, Japan) were used. 
The DC-bus capacitance was built with four $470 \mu \mathrm{F} / 450 \mathrm{~V}$ capacitors, to reach up to $900 \mathrm{~V}$. The current sensing was implemented using the Hall effect sensors LTS 15-NP (LEM, Geneva, Switzerland) and the grid voltage and DC-voltage sensing using the LV25-P (LEM, Geneva, Switzerland). The LCL filter used at the inverter side is specified in Table 1. The grid-connected inverter including the PI with grid-voltage feedforward or the PR controller is shown in Figure 10.

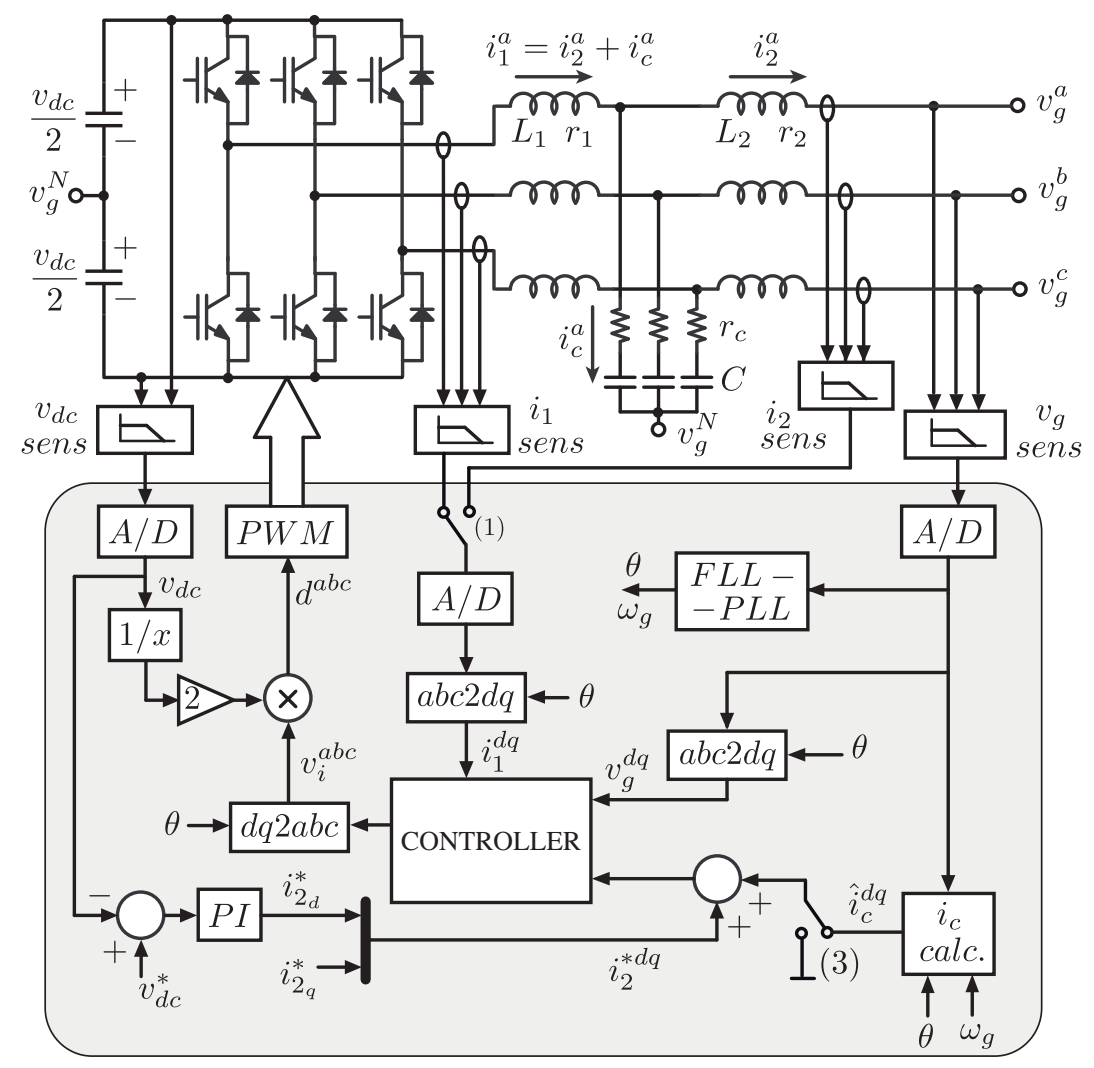

Figure 10. Transformerless three-phase grid-connected inverter with the LCL filter, current controller and CE strategy.

The control was programmed in the Renesas floating point microcontroller R5F5630EDDFB (Renesas Electronics Corporation, Tokyo, Japan). A sampling frequency of $20 \mathrm{kHz}$ was used with a center-aligned PWM pattern. The grid synchronization was carried out by means of an FLL-PLL [23]. The FLL gives an accurate measure of the grid frequency $\omega_{g}$, which is used in (13) and (15), and to adapt the resonant frequencies $\omega_{h}$ of the PR control in (7).

The programmed PI and PR control parameters are listed in Table 2. The proportional gain $k_{p} \approx L \omega_{c}=6.71 \Omega$ sets the bandwidth, and the integral gain $k_{i}=k_{p} \omega_{z}=2530 \Omega \cdot \mathrm{s}^{-1}$ sets the PI's zero $\omega_{z}$ one decade below the bandwidth. The resonant compensators were tuned experimentally. Starting from the PI configuration, the power spectral density of the grid current $i_{2}$ was measured, identifying the following dominant harmonics: 3rd, 7th, 13th and 15th (positive sequence) and 5th, 11th and 17th (negative sequence). To cancel the seventh and 13th positive sequence harmonics, resonant compensators in the $d q$ frame for $h=\{6,12\}$ are required, which in turn cancel the negative sequence fifth and 11th [24]. Another resonant was added for $h=2$ to cancel the third harmonic and to remove any fundamental negative sequence.

In order to study the group delay of the used PI control with the CCF scheme, Figure 11 shows the experimental closed-loop response of the system. These results were obtained by programming the current references: 


$$
\begin{aligned}
& i_{d}^{*}=I_{0} \cdot \cos \left(\theta_{0}\right)+I \cdot \cos \left[h \cdot\left(\theta+\frac{\pi}{2}\right)\right] \\
& i_{q}^{*}=-I_{0} \cdot \sin \left(\theta_{0}\right)+I \cdot \sin \left[h \cdot\left(\theta+\frac{\pi}{2}\right)\right]
\end{aligned}
$$

with $I_{0}=5 \mathrm{~A}, I=3 \mathrm{~A}$ and sweeping for $h=\{1,2, \ldots, 49\}$. The amplitude and phase $\phi$ of the resulting harmonic on $i_{1}$ were measured using the Power \& Quality Analyzer CA8384B from Chauvin Arnoux (Paris, France). In order to get delay measurements $\phi$ referred to the reference harmonic (16), which is in phase with the grid voltage, both fundamental voltage and current have to be perfectly in phase. This is achieved by adjusting the appropriate value of $\theta_{0}$ before starting the measurements. The group delay was approximated by $G_{d}(h)=\frac{-\phi}{\omega_{g} h T}$ samples, being $\phi$ the measured closed-loop phase in radians. A group delay between two and five samples has been experimentally obtained.
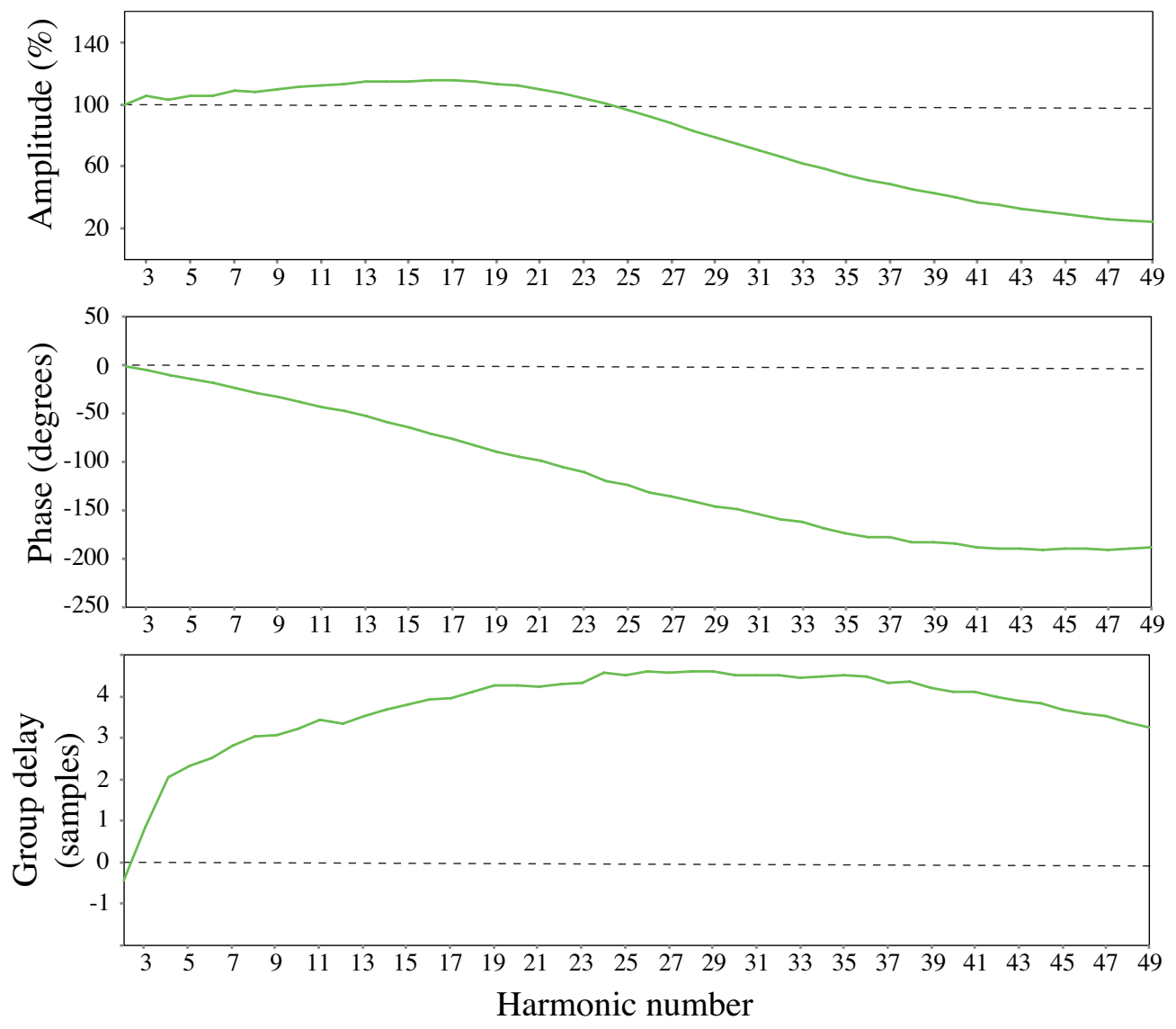

Figure 11. Experimental closed-loop frequency response and group delay for the CCF scheme with PI and grid-voltage feedforward cancellation.

Figure $12 \mathrm{a}, \mathrm{b}$ is intended to compare the line current quality obtained with the proposed CCF $+\mathrm{CE}$ technique and the GCF technique. Figure 12a shows the grid current waveform and its spectral density at full active power with GCF, giving a THD around $1.3 \%$. Figure $12 \mathrm{~b}$ shows the grid current waveform and its spectral density at full active power with $\mathrm{CCF}+\mathrm{CE}$, giving a THD around $0.7 \%$. A reduction of THD higher than the $45 \%$ is achieved.

Hereafter, the experimental results are focused on the performance of the CCF scheme with PI control and grid-voltage feedforward. 

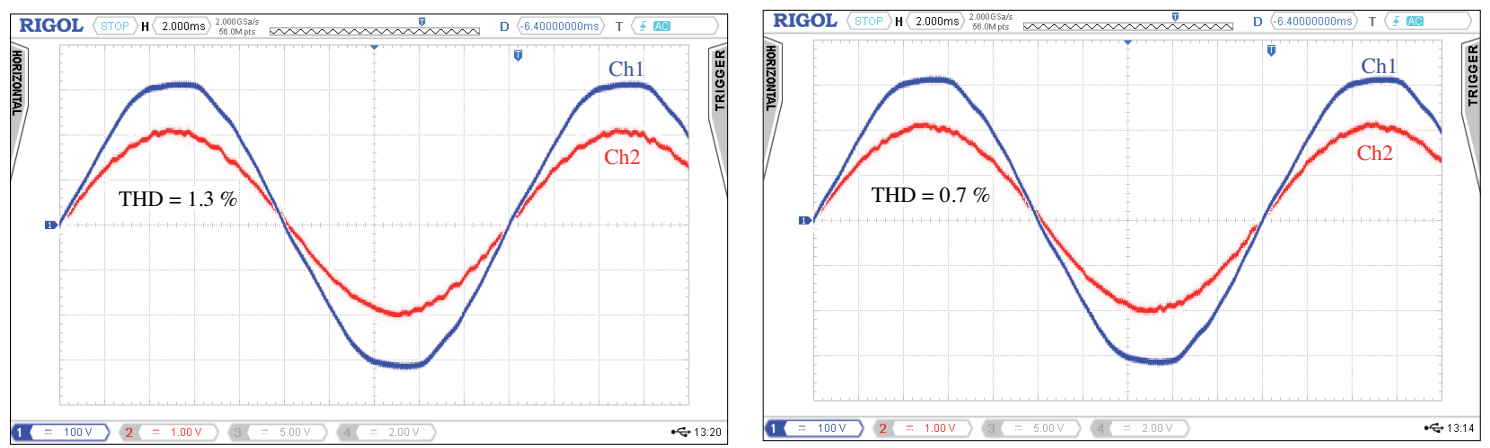

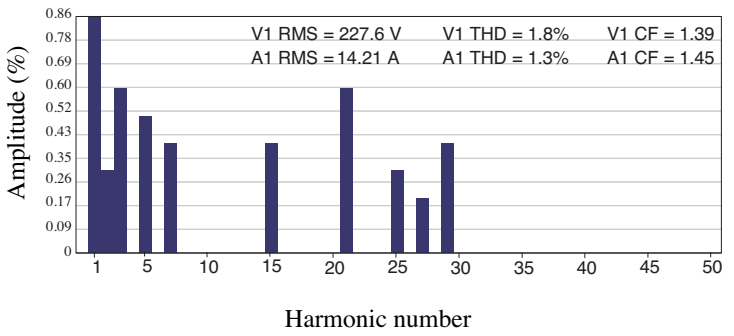

(a)

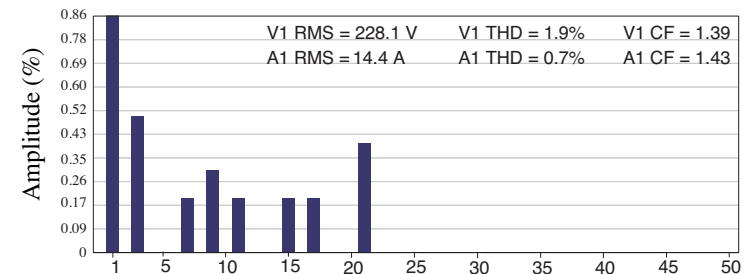

Harmonic number

(b)

Figure 12. Grid current and harmonic content at nominal $10 \mathrm{kVA}$ using the $\mathrm{CE}$ technique in a CCF with PI control and grid-voltage feedforward (a) and GCF with PR control (b). Ch1: grid voltage (100 V/div). Ch2: grid current (10 A/div).

Figure 13 gives the line current THD variation with the number of estimation leading samples when using the CCF and the $\mathrm{CE}$ technique, showing that an optimal delay compensation is achieved with six leading samples for all currents levels, i.e., $n_{f}=6$ will be considered in Equation (15).

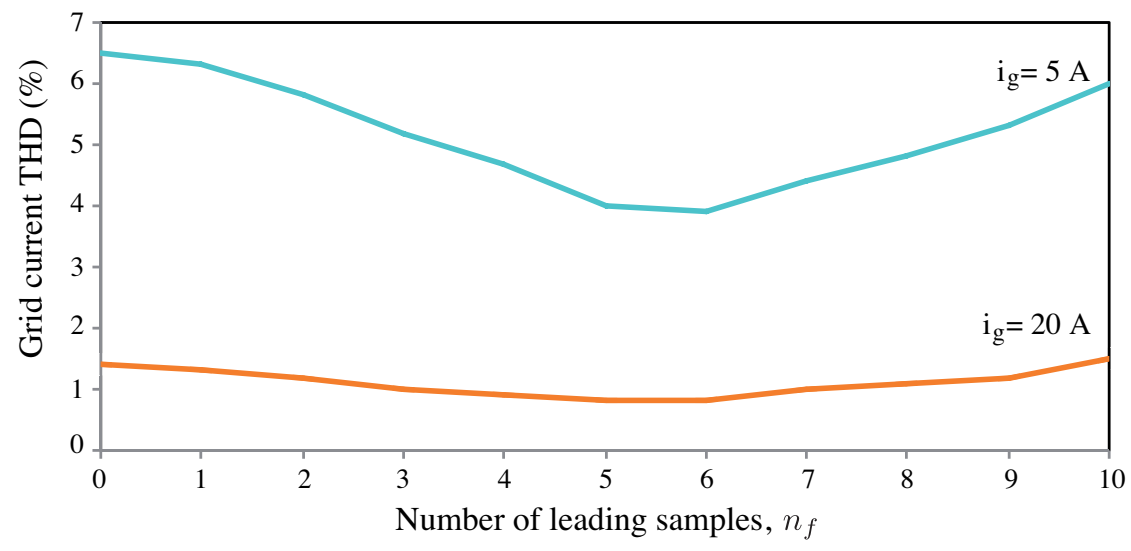

Figure 13. Grid current THD as a function of the estimation leading samples $\left(n_{f}\right)$ using the CCF schema and the $\mathrm{CE}$ technique.

Figure 14 illustrates the effect of using or not the CE strategy. At half the nominal power, the grid current THD is $4.2 \%$ without CE, and it reduces to $1.5 \%$ with CE. A reduction of THD around $64 \%$ is achieved.

Finally, in order to present a full comparative study, Figure 15 summarizes the grid current THD for the CCF scheme with and without the CE technique and the GCF scheme with a PR control. Although the GCF achieves very low THD levels around 1.3\% at nominal power, better than the CCF with no $\mathrm{CE}$ ( $1.9 \%$ at nominal power), the bets results are obtained with the proposed $\mathrm{CE}$ technique in $\mathrm{CCF}$, which lowers the THD down to $0.7 \%$. 


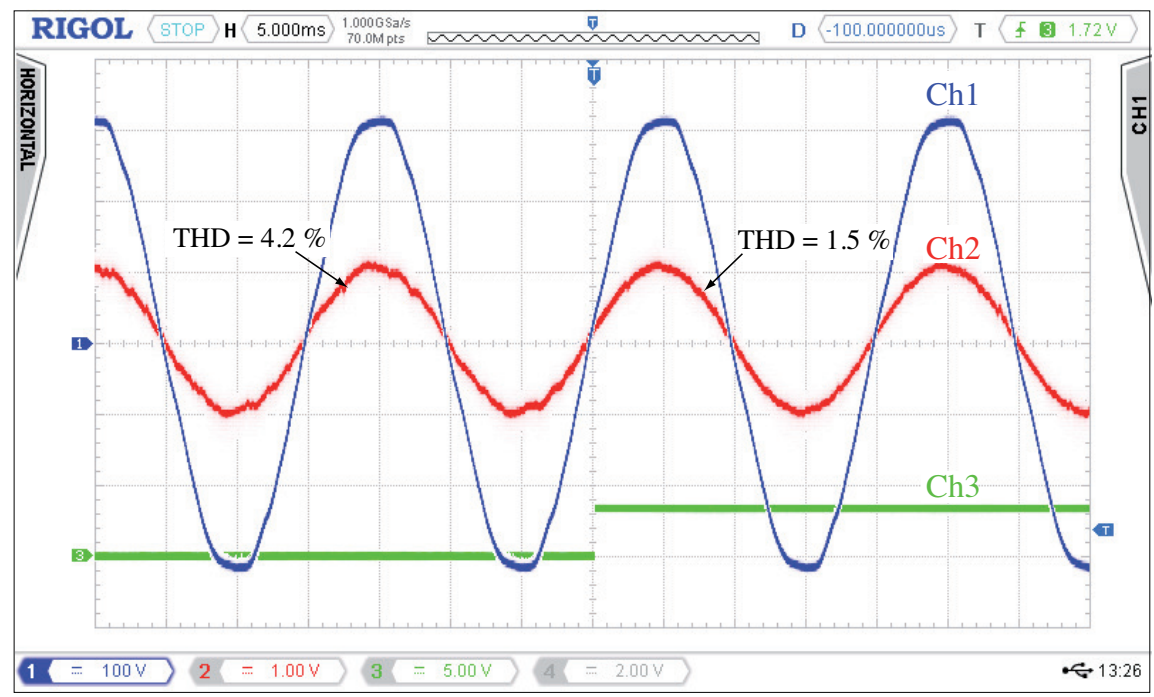

Figure 14. Capacitive Emulation (CE) activation-deactivation with the CCF scheme and PI control with grid-voltage feedforward cancellation at half nominal power. Ch1: grid voltage (100 V/div). Ch2: grid current $(10 \mathrm{~A} / \mathrm{div})$. Ch3: CE on/off signal $(0=$ off, $1=$ on $)$.

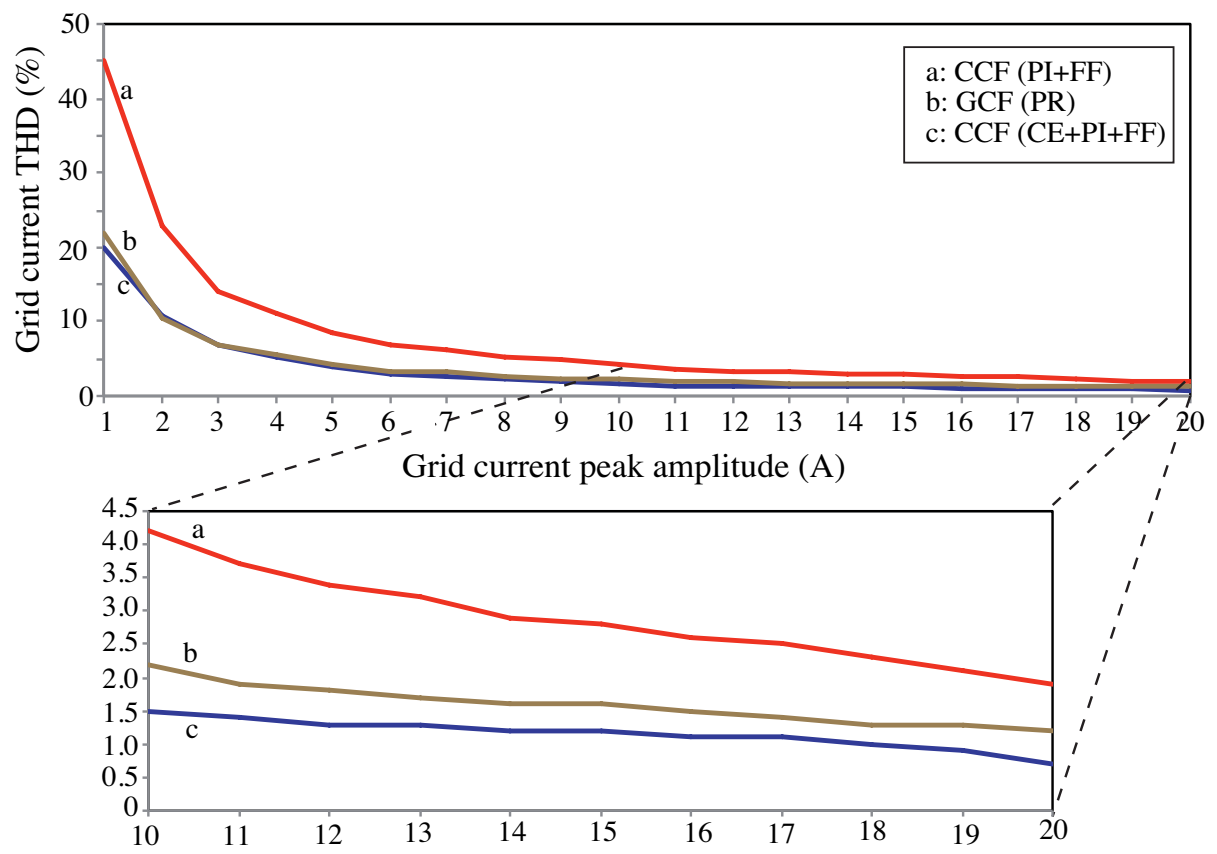

Figure 15. Grid current THD for CCF (with and without the CE technique) and the GCF strategy with a PR control.

\section{Conclusions}

This paper presents a capacitive emulation (CE) technique that significantly reduces the harmonic distortion of the line currents when using LCL filters and converter current feedback (CCF). The proposed CE method consists of generating the grid-induced capacitive current harmonics with the inverter, which requires an estimation of the capacitive current disturbance to be fed forward as the current reference. This paper proposes a simple constant-size buffer-based method to get an ahead filtered disturbance estimation that compensates for the control delay. The filtering method is non-distorting, adapts to grid voltage amplitude and frequency variations and avoids peak currents when a grid voltage sag occurs. 
The effectiveness of the CE method has been proven experimentally on a 10-kVA transformerless LCL-filtered inverter with PI control and grid-voltage feedforward. Experimental results show a line current distortion reduction of more than $45 \%$ compared with a GCF schema with multi-resonant PR control.

Author Contributions: Jose Miguel Espi proposed the main idea and performed the theoretical analysis, and Rafael Garcia-Gil and Jaime Castello contributed to the development and discussion of the results. All authors have contributed to the practical implementation, have performed the experiments and wrote the paper. All authors have read and approved the final manuscript.

Conflicts of Interest: The authors declare no conflict of interest.

\section{References}

1. Lorzadeh, I.; Askarian Abyaneh, H.; Savaghebi, M.; Bakhshai, A.; Guerrero, J.M. Capacitor current feedback-based active resonance damping strategies for digitally-controlled inductive-capacitiveinductive-filtered grid-connected inverters. Energies 2016, 9, 642.

2. Tang, Y.; Loh, P.C.; Wang, P.; Choo, F.H.; Gao, F. Exploring inherent damping characteristic of LCL-filters for three-phase grid-connected voltage source inverters. IEEE Trans. Power Electron. 2012, 27, 1433-1443.

3. Said-Romdhane, M.B.; Naouar, M.W.; Belkhodja, I.S.; Monmasson, E. An improved LCL filter design in order to ensure stability without damping and despite large grid impedance variations. Energies 2017, 10, 336.

4. Yu, Y.; Li, H.; Li, Z.; Zhao, Z. Modeling and analysis of resonance in LCL-type grid-connected inverters under different control schemes. Energies 2017, 10, 104.

5. Pan, D.; Ruan, X.; Wang, X.; Yu, H.; Xing, Z. Analysis and design of current control schemes for LCL-type grid-connected inverter based on a general mathematical model. IEEE Trans. Power Electron. 2017, 32, 4395-4410.

6. Wang, J.; Yan, J.D.; Jiang, L. Pseudo-derivative-feedback current control for three-phase grid-connected inverters with lcl filters. IEEE Trans. Power Electron. 2016, 31, 3898-3912.

7. Wang, J.; Yan, J.D.; Jiang, L.; Zou, J. Delay-dependent stability of single-loop controlled grid-connected inverters with LCL filters. IEEE Trans. Power Electron. 2016, 31, 743-757.

8. Zhang, X.; Spencer, J.; Guerrero, J. Small-signal modeling of digitally controlled grid-connected inverters with LCL filters. IEEE Trans. Ind. Electron. 2013, 60, 3752-3765.

9. Jia, Y.; Zhao, J.; Fu, X. Direct grid current control of LCL-filtered grid-connected inverter mitigating grid voltage disturbance. IEEE Trans. Power Electron. 2014, 29, 1532-1541.

10. Bao, C.; Ruan, X.; Wang, X.; Li, W.; Pan, D.; Weng, K. Step-by-step controller design for LCL-type grid-connected inverter with capacitor-current-feedback active-damping. IEEE Trans. Power Electron. 2014, 29, 1239-1253.

11. Meyer, R.; Zlotnik, A.; Mertens, A. Fault ride-through control of medium-voltage converters with LCL filter in distributed generation systems. IEEE Trans. Ind. Appl. 2014, 50, 3448-3456.

12. Dannehl, J.; Wessels, C.; Fuchs, F. Limitations of voltage-oriented PI current control of grid-connected PWM rectifiers with LCL filters. IEEE Trans. Ind. Electron. 2009, 56, 380-388.

13. Ghoshal, A.; John, V. Active damping of LCL filter at low switching to resonance frequency ratio. IET Power Electron. 2015, 8, 574-582.

14. Citro, C.; Siano, P.; Cecati, C. Designing inverters' current controllers with resonance frequencies cancellation. IEEE Trans. Ind. Electron. 2016, 63, 3072-3080.

15. Jin, W.; Li, Y.; Sun, G.; Bu, L. H repetitive control based on active damping with reduced computation delay for LCL-type grid-connected inverters. Energies 2017, 10, 586.

16. Wang, X.; Blaabjerg, F.; Loh, P.C. Grid-current-feedback active damping for LCL resonance in grid-connected voltage-source converters. IEEE Trans. Power Electron. 2016, 31, 213-223.

17. Xin, Z.; Loh, P.C.; Wang, X.; Blaabjerg, F.; Tang, Y. Highly accurate derivatives for LCL-filtered grid converter with capacitor voltage active damping. IEEE Trans. Power Electron. 2016, 31, 3612-3625.

18. Xin, Z.; Wang, X.; Loh, P.C.; Blaabjerg, F. Grid-current-feedback control for LCL-filtered grid converters with enhanced stability. IEEE Trans. Power Electron. 2017, 32, 3216-3228.

19. Zhang, N.; Tang, H.; Yao, C. A systematic method for designing a PR controller and active damping of the LCL filter for single-phase grid-connected pv inverters. Energies 2014, 7, 3934-3954. 
20. Gonzalez-Espin, F.; Garcera, G.; Patrao, I.; Figueres, E. An adaptive control system for three-phase photovoltaic inverters working in a polluted and variable frequency electric grid. IEEE Trans. Power Electron. 2012, 27, 4248-4261.

21. Castello, J.; Espi, J.M.; Garcia-Gil, R. A new generalized robust predictive current control for grid-connected inverters compensates anti-aliasing filters delay. IEEE Trans. Ind. Electron. 2015, 63, 4485-4494.

22. Yan, Q.; Wu, X.; Yuan, X.; Geng, Y. An improved grid-voltage feedforward strategy for high-power three-phase grid-connected inverters based on the simplified repetitive predictor. IEEE Trans. Power Electron. 2016, 31, 3880-3897.

23. Rodriguez, P.; Luna, A.; Candela, I.; Mujal, R.; Teodorescu, R.; Blaabjerg, F. Multiresonant frequency-locked loop for grid synchronization of power converters under distorted grid conditions. IEEE Trans. Ind. Electron. 2011, 58, 127-138.

24. Liserre, M.; Teodorescu, R.; Blaabjerg, F. Multiple harmonics control for three-phase grid converter systems with the use of PI-RES current controller in a rotating frame. IEEE Trans. Power Electron. 2006, 21, 836-841.

(C) 2017 by the authors. Licensee MDPI, Basel, Switzerland. This article is an open access article distributed under the terms and conditions of the Creative Commons Attribution (CC BY) license (http:/ / creativecommons.org/licenses/by/4.0/). 